\title{
Surface and Flow Field Measurements on the FAITH Hill Model
}

\author{
James H. Bell, ${ }^{1}$ James T. Heineck, ${ }^{2}$ Gregory Zilliac, ${ }^{3}$ Rabindra D. Mehta ${ }^{4}$ \\ Experimental Aero-Physics Branch, NASA Ames Research Center, Moffett Field, CA, 94035, USA \\ Kurtis R. Long $^{5}$ \\ AerospaceComputing, Inc., Mountain View, CA, 94035, USA
}

\begin{abstract}
A series of experimental tests, using both qualitative and quantitative techniques, were conducted to characterize both surface and off-surface flow characteristics of an axisymmetric, modified-cosine-shaped, wall-mounted hill named "FAITH" (Fundamental Aero Investigates The Hill). Two separate models were employed: a 6" high, 18" base diameter machined aluminum model that was used for wind tunnel tests and a smaller scale (2" high, 6" base diameter) sintered nylon version that was used in the water channel facility. Wind tunnel and water channel tests were conducted at mean test section speeds of 165 fps (Reynolds Number based on height $=\mathbf{5 0 0 , 0 0 0}$ ) and 0.1 fps (Reynolds Number of 1000), respectively. The ratio of model height to boundary later height was approximately 3 for both tests. Qualitative techniques that were employed to characterize the complex flow included surface oil flow visualization for the wind tunnel tests, and dye injection for the water channel tests. Quantitative techniques that were employed to characterize the flow included Cobra Probe to determine point-wise steady and unsteady 3D velocities, Particle Image Velocimetry (PIV) to determine 3D velocities and turbulence statistics along specified planes, Pressure Sensitive Paint (PSP) to determine mean surface pressures, and Fringe Imaging Skin Friction (FISF) to determine surface skin friction (magnitude and direction). This initial report summarizes the experimental set-up, techniques used, data acquired and describes some details of the dataset that is being constructed for use by other researchers, especially the CFD community. Subsequent reports will discuss the data and their interpretation in more detail.
\end{abstract}

\section{Nomenclature}

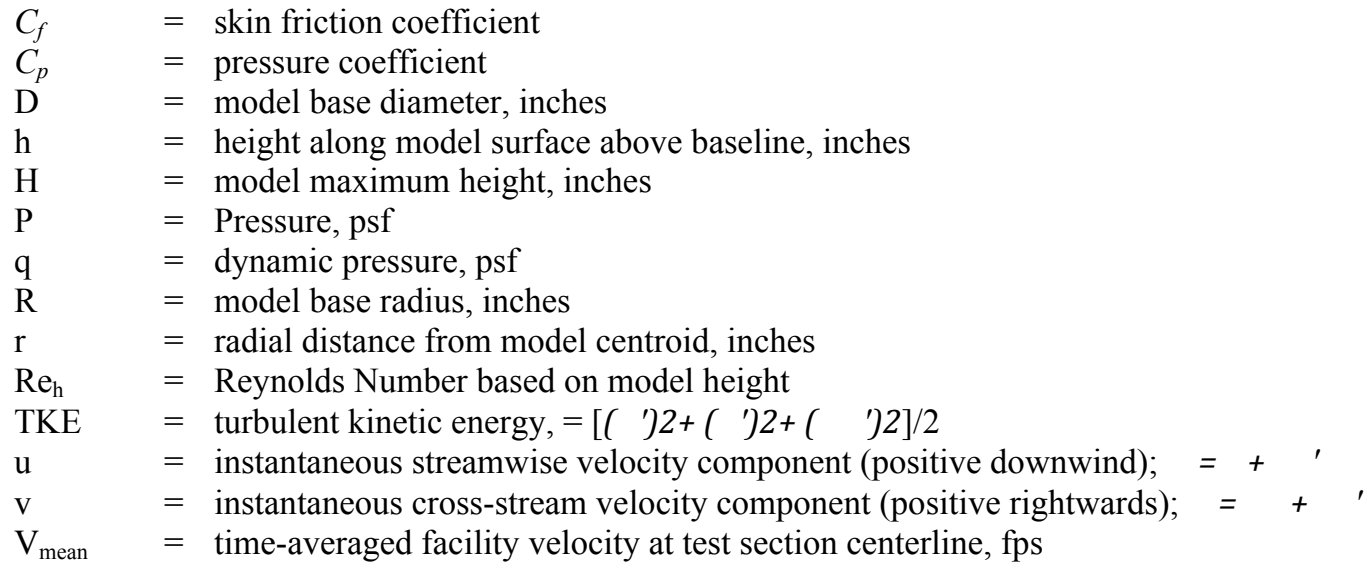

\footnotetext{
${ }^{1}$ Aerospace Engineer; james.h.bell@nasa.gov; AIAA Member

${ }^{2}$ Photo Technologist; james.t.heineck@nasa.gov; AIAA Member

${ }^{3}$ Aerospace Engineer; greg.zilliac@nasa.gov; AIAA Member

${ }^{4}$ Branch Chief; rabindra.d.mehta@nasa.gov; AIAA Associate Fellow

${ }^{5}$ Senior Research Engineer; kurtis.r.long@nasa.gov.
} 
$=$ time-averaged streamwise velocity component (positive downwind)

$=$ time-averaged cross-stream velocity component (positive rightwards)

$=$ time-averaged vertical velocity component (positive upwards)

$=$ unsteady streamwise velocity component (positive downwind)

, $\quad=$ unsteady cross-stream velocity component (positive rightwards)

, $\quad=$ unsteady vertical velocity component (positive upwards)

$\mathrm{x}=$ distance downstream of model centroid, inches

$\mathrm{y} \quad=$ distance right of model centroid, inches

$\mathrm{Z}=$ distance above tunnel floor, inches

$\delta=$ boundary layer height, inches

\section{Introduction}

Subsonic turbulent boundary layer flow around a sufficiently steep axisymmetric bump or hill (figure 1) creates a variety of interesting on- and off-surface flow features, including unsteady vortical flow separation and reattachment. The ability to characterize such features is of fundamental importance for a variety of design optimization and analysis situations. Previous experimental studies (references 1 through 9) investigated the wake characteristics of an axisymmetric hill immersed in a subsonic turbulent boundary layer. References 6 through 9 used a variety of experimental techniques, including Laser Doppler Velocimetry (LDV), Hot-Wire Anemometry (HWA), and piezoresistive pressure transducers, to investigate both on- and off-surface parameters associated with the separated flow arising from an axisymmetric hill immersed in a turbulent boundary layer flow. In 2006, the turbulence modeling and simulation community (reference 10) set the axisymmetric hill or bump immersed in a subsonic boundary layer as a test case worthy of further computational study. As a result, several computational large eddy simulation (LES) investigations (references 11 through 14) used these experimental studies to help assess the capabilities of various computational techniques that might be employed to help gain insight into the physical wake phenomena associated with separating and reattaching flows about a wall-mounted axisymmetric hill immersed in a subsonic boundary layer.

The objective of this project was to conduct a related set of wind tunnel and water channel experiments on a small wind tunnel model known as FAITH (Fundamental Aero Investigates The Hill; figure 2) that would contribute towards understanding of the separating and reattaching wake characteristics associated with a wall-mounted axisymmetric hill immersed in a subsonic boundary layer. This project employed a battery of experimental techniques to measure significant on-and off-surface parameters, along with well-documented inlet and outlet flow conditions, with the goal of establishing an experimental database that will enable assessment and optimization of the ability of computational methods to predict the complex flow behavior in the separated and reattaching regions downstream of an axisymmetric hill immersed in a subsonic boundary layer. The project has produced quantitative and qualitative wake data from seven different measurement techniques, including Particle Image Velocimetry (PIV), Pressure Sensitive Paint (PSP), Fringe Imaging Skin Friction (FISF), unsteady 3D velocity measurement, mean surface pressure, surface oil flow visualization, and water channel dye flow visualization. Table I summarizes the overall effort.

Table I FAITH Project Summary

\begin{tabular}{|c|c|c|c|c|c|c|}
\hline $\begin{array}{l}\text { Model and } \\
\text { Size }\end{array}$ & $\begin{array}{l}\text { Vmean } \\
\text { (fps); } \\
\operatorname{Re}_{\mathrm{h}}\end{array}$ & $\mathrm{H} / \delta$ & Test Technique & $\begin{array}{l}\text { Measurements/ } \\
\text { Documentation }\end{array}$ & $\begin{array}{l}\text { Measurement Location } \\
\text { Description } \\
\text { (Center of Model Base: } \\
\mathrm{X}=\mathrm{Y}=\mathrm{Z}=0 \text { ) }\end{array}$ & Measurement Extents \\
\hline $\begin{array}{l}\text { SLS Nylon; } \\
\text { R =3" } \\
\mathrm{H}=2 "\end{array}$ & $\begin{array}{l}0.1 \\
3 \mathrm{E} 3\end{array}$ & 3 & $\begin{array}{c}\text { Water Channel Dye Flow } \\
\text { Visualization }\end{array}$ & Video, Still Images & $\begin{array}{c}\text { Overall Flowfield } \\
\text { Centerline Laser Sheet }\end{array}$ & $\begin{array}{l}0<\mathrm{x} / \mathrm{H}<6 ; \mathrm{y}=0,0<\mathrm{z} / \mathrm{H}<2 \\
0<\mathrm{x} / \mathrm{H}<6 ; \mathrm{y}=0,0<\mathrm{z} / \mathrm{H}<2\end{array}$ \\
\hline \multirow{5}{*}{$\begin{array}{c}\text { aluminum; } \\
\mathrm{R}=9 " \\
\mathrm{H}=6 ”\end{array}$} & \multirow[t]{5}{*}{$\begin{array}{l}160 \\
5 \mathrm{E} 5\end{array}$} & \multirow[t]{5}{*}{3} & $\begin{array}{c}\text { Wind Tunnel Oil Flow } \\
\text { Visualization }\end{array}$ & Still Images & Entire Surface of Model & $0<\mathrm{x} / \mathrm{H}<6 ; \mathrm{y}=0,0<\mathrm{z} / \mathrm{H}<2$ \\
\hline & & & PSP & Mean Surface Pressures & Entire Surface of Model & $\mathrm{r} / \mathrm{R}<1$ \\
\hline & & & PIV & $\begin{array}{l}4000 \text { samples@ } 2 \text { hz; } \\
\text { 3D Velocity Statistics }\end{array}$ & 8 Longitudinal-Vertical planes & $\begin{array}{c}0<\mathrm{x} / \mathrm{H}<6 ; \mathrm{y}=--2,0 \\
2,3,4,4.75,6,7 ; 0<\mathrm{z} / \mathrm{H}<2\end{array}$ \\
\hline & & & Cobra Probe & $\begin{array}{c}7 \text { sec samples @ } 1250 \text { hz of } \\
\text { 3D velocity data; }\end{array}$ & $\begin{array}{c}1 \text { centerline plane ( } 500 \text { points) } \\
7 \text { vertical-lateral planes ( } 500 \\
\text { points) }\end{array}$ & $\begin{array}{l}0<\mathrm{x} / \mathrm{H}<6 ; \mathrm{y}=0,0<\mathrm{z} / \mathrm{H}<2 \\
0<\mathrm{x} / \mathrm{H}<6 ; \mathrm{y}=0,0<\mathrm{z} / \mathrm{H}<2\end{array}$ \\
\hline & & & FISF & Mean_Skin Frietion & Entire-Sufface of Model & \\
\hline
\end{tabular}




\begin{tabular}{|l|l|l|l|c|c|c|}
\hline & & & Magnitude and Direction & & \\
\cline { 3 - 6 } & & & Discrete Surface Pressures & Mean Surface Pressures & Entire Surface of Model & 12 discrete locations \\
\hline
\end{tabular}

Measurement techniques that are still in progress include unsteady discrete surface pressure measurements (Kulites) and some additional planes of PIV data. This initial report summarizes the data acquired to this point, and also describes the dataset that is being made available for use by other researchers. Subsequent reports will discuss the data in more detail.

\section{Facilities and Models}

Wind Tunnel Facility. The NASA Ames Fluid Mechanics Lab's 48" wide x 32" tall x 120" long indraft facility (figure 3) was employed for this effort. It is an open-circuit, sonic throat, indraft wind tunnel. The facility centrifugal compressor runs at constant speed; mass flow (and hence, speed) through the test section is controlled by means of a variable-area sonic throat. Flow travels from the lab into the bellmouth, through a honeycomb and 3 screens, and into a 9:1 contraction. Downstream of the contraction is the wind tunnel test section, which is approximately 48 " wide, 32 " tall, and 120 " long. The design centerline mean velocity in the test section is $170 \mathrm{fps}$, corresponding to a Reynolds Number of approximately 1.1 million per foot. The freestream turbulence intensity $(\mathrm{TI})$ and boundary layer height $(\delta)$ at the midpoint of the empty test section are approximately $0.13 \%$ and 1.5 ", respectively.

Wind Tunnel Facility Control Instrumentation. During wind tunnel testing, existing facility instrumentation was used for control and speed measurement. MKS Instruments 698A Barotron differential pressure transducers were employed to measure inlet and test section dynamic pressures, and a United Sensor pitot-static thermocouple was employed to measure test section dynamic pressure and temperature, which were used in speed computations. The FML's standard Labview-based wind tunnel instrumentation system (BDAS) was employed to control tunnel conditions and record data for the wind tunnel portion of the project. The BDAS and MKS transducers were calibrated in-situ within one month prior to each test.

Water Channel Facility. The NASA Ames Fluid Mechanics Lab's 16" wide x 8" deep x 96" long water channel facility (figure 4) was employed for this effort. It is a closed-circuit, open channel facility. The facility pump runs at a user-selectable variable speed, to control mass flow and hence, test section speed. Flow travels through a honeycomb and three screens into the water channel test section, which is approximately 16 " wide, 8" deep, and 96" long. The design velocity in the test section is $0.1 \mathrm{fps}$, corresponding to a Reynolds Number of approximately 3000 per foot. The freestream turbulence level and boundary layer height at the midpoint of the empty test section are approximately $1 \%$ and 3", respectively. A three inch tall boundary layer splitter plate was used to control boundary layer thickness; the model was positioned at various locations along the plate to produce different values of boundary layer thickness.

Models. Two separate axisymmetric, modified-cosine-shaped, wall-mounted models were employed for this effort. For the wind tunnel tests, a 6 " high $(\mathrm{H}=6 "), 18$ " base $(\mathrm{R}=9 ")$ diameter machined aluminum model was used. For the water channel tests, a smaller scale $(\mathrm{H}=2$ ", $\mathrm{R}=6$ ") sintered nylon version of the wind tunnel model was used. For the large model, the height above ground (h) varied with radial distance from the centroid (r) by

$$
h=3 \cdot \operatorname{Cos}\left(\frac{\pi}{9} r\right)+3
$$

To minimize chances of model edge damage and personnel injury, the thin, sharp model edges were blunted slightly by sanding. Both models were polished to a smooth finish. To facilitate flow visualization sequences, each model was painted flat black. For the FISF sequence, the large model was nickel-plated to a highly reflective smooth mirrored finish.

\section{Test Equipment and Procedures}

For each wind tunnel test, the aluminum model was mounted in the middle of the test section, along the centerline, and the tunnel was run at 165 fps. For each water channel test, the sintered nylon model was mounted in 
the middle of the test section, along the centerline, and the channel was run at $0.1 \mathrm{fps}$. Each technique and test specific equipment is summarized below.

Cobra Probe Wind Tunnel Tests and Equipment. The Cobra probe, made by Turbulent Flow Instrumentation (TFI), Ltd, is a 4 hole pressure probe used to determine unsteady 3D components of velocity at frequencies less than 10 $\mathrm{kHz}$. The Cobra probe employs Honeywell $2.5 \mathrm{kPa}$ transducers to measure flow speeds up to $60 \mathrm{~m} / \mathrm{s}$, and has a cone of acceptance of $\pm 45 \mathrm{deg}$, centered on the probe centerline. The probe was connected to the facility data collection and control computer via National Instruments A/D card. Prior to Cobra Probe test sequences, the model was attached to the wind tunnel floor along the test section centerline, and the grid of desired measurement locations was coded into the traverse control module of the data collection system. The wind tunnel was operated at the desired test speed, and the data collection routine was started. The 3-axis traverse, mounted above the test section in a constant pressure plenum moved a single Cobra probe to each of the 3D spatial locations, and acquired 6 seconds of $1250 \mathrm{hz}$ pressure data at each location. Simultaneously, the Cobra probe software computed 3D velocity components for each measurement.

PSP Wind Tunnel Tests. Test-specific instrumentation used during PSP sequences included Light Emitting Diode (LED) Ultraviolet (UV) illumination lamps mounted in the wind tunnel ceiling, imaging cameras, and data collection software running on a PC laptop computer. Prior to PSP test sequences, the model was covered with a white base coat, followed by Pressure Sensitive Paint. The model was attached to the wind tunnel floor along the test section centerline, and the wind tunnel was operated at the desired test speed. Several UV LED lamps, mounted directly above the model in the test section ceiling were used to illuminate the model. To achieve temperature equilibrium of the model, PSP, and wind, the model was run for approximately 1 hour at $50 \mathrm{fps}$, then 30 minutes at $100 \mathrm{fps}$, and then 15 minutes at the desired test speed of $160 \mathrm{fps}$. A camera mounted directly above the model in the test section ceiling was employed to acquire 50 separate images and then another 15 images with the UV lamps off as a reference. The tunnel was stopped, and an additional 50 images were acquired while the UV lamps remained on. The wind off and UV lamp-off data were employed as a baseline for the wind-on images, to enable computation of mean surface pressures.

PIV Wind Tunnel Tests. The PIV data were acquired using a dual cavity, pulsed Nd:YAG laser with 350 millijoules per pulse and two 11 Megapixel PIV cameras with the frame straddling function. The plane of the laser was projected through the aft test section side wall to a vertical mirror mounted in the aft portion of the test section. This reflected the laser sheet forward, along the centerline of the test section in the vertical stream-wise plane. The two cameras were placed outside the test section, upstream of the model, and viewed the laser light in the forward-scatter mode. The image field of view captured from the top of the Hill to $650 \mathrm{~mm}$ (24 inches) downstream, from the floor to $250 \mathrm{~mm}$ (10 inches). The model was moved laterally in the test section to acquire planes of data along different span-wise positions of the wake. Planes of data were acquired at -2, 0 (centerline), 2, 3, 4, 4.75, 6, and 7 inches.

The system operated at $2 \mathrm{~Hz}$ and 4000 samples were acquired. The data were reduced using LaVision DaVis v8 software. The region of interest was processed using 64 x 64 pixel interrogation windows, with $75 \%$ overlap. The software uses a grid distortion algorithm that renders velocity gradients that are smaller than the interrogation window. The 4000 samples were averaged with statistics derived from that average. Prior to PIV sequences, the model and test section interior were painted flat black to minimize unwanted reflections. The wind tunnel was run at the desired test speed, theatrical fog was introduced across the test section inlet, and the fog particles filled the test section and centerline laser sheet, facilitating data capture.

FISF Wind Tunnel Tests. Test-specific instrumentation used during FISF sequences included oil, a digital SLR camera, and data collection and vector processing software running on a PC laptop computer. Prior to FISF sequences, the model was plated with a Nickel coating to produce maximum reflectivity. The model was mounted onto the wind tunnel floor along the test section centerline. Oil drops of a known viscosity were then applied to the surface of the model. The wind tunnel was then run at the desired test speed. The wind caused the oil droplets to move in the direction of the surface shear stress. The tunnel was stopped, and a bright light source was applied to the oil-streaked model, which created an optical fringe pattern on the model. Photos were taken of the model, and the direction and spacing of the resultant fringe patterns were used to determine local skin friction vectors. 
Discrete Surface Pressure Measurement Wind Tunnel Tests. Prior to the discrete surface pressure measurements, 12 different pressure port holes were drilled in the model at various locations. The model was attached to the wind tunnel floor along the test section centerline, and the pressure ports were connected via tubing to a PSI 8400 data collection system. The wind tunnel was operated at a series of speeds between 0 and $170 \mathrm{fps}$ in $10 \mathrm{fps}$ increments, and the data collection routine was operated for each speed, acquiring 6 seconds of pressure data at each speed.

Oil Flow Visualization Wind Tunnel Tests. During oil flow visualization sequences, a mixture of motor oil, oleic acid, and artist pigment was mixed together, filtered, and then applied to the model. Prior to oil flow visualization tests, the model was painted black and then attached to the wind tunnel floor. The model and the floor downstream of the model were then coated with a mixture of motor oil, artists pigment, and oleic acid. The tunnel was operated to the desired speed. The air caused the pigmented oil to flow over the model and floor surface, setting up various patterns. The tunnel was allowed to run at the same velocity for approximately 10 minutes, to ensure that the flow patterns achieved a stable location. The tunnel was stopped, and photographs were taken of the resultant oil patterns.

Dye Flow Water Channel Tests. During water channel flow visualization sequences, a fluorescent dye solution was injected via custom apparatus and illuminated by four UV lamps. Prior to dye flow visualization tests, the model was attached to the water channel floor. The water channel was started, and yellow-green dye was injected upstream of the model via a dye injection apparatus, while red dye was injected downstream of the model, within the separated flow region of the model. Photographs and videos were taken of the resultant flow patterns.

\section{Results}

Subsequent reports will include detailed analysis of all physical features associated with this flow; at the present time, the authors here attempt only to provide a broad overview of the data that have been acquired and added to the database. Summaries of the data acquired so far are provided below.

a) Water Channel Flow Visualization Tests. Figures 5 through 7 were taken during the water channel dye flow experiments. Figures 5 and 6 illustrate the wake of the hill, and show particularly well the line of separation on the backside of the model. Figure 6 depicts several upwind flow features, including the small necklace vortex at the base of the hill, while figure 7, which was taken by illuminating the wake with a thin sheet of laser light, shows the complicated flow patterns present in the separated wake.

b) Wind Tunnel Oil Flow Visualization Tests. Figures 8 and 9 were taken during the wind tunnel oil flow experiments. Figures 8 and 9 both show the line of separation on the backside of the model, along with the location of reattachment, and even the footprint of a small necklace vortex, which trails back along either side of the base. Figure 9 depicts separated, reverse, and low speed separated flow zones via various pigmented oils.

c) Wind Tunnel Pressure Sensitive Paint (PSP) Tests. Figure 10 depicts mean surface pressure coefficient data acquired during PSP tests. Figure 10 shows the existence of a large high pressure region on the upwind side of the hill, as well as the presence of a large low pressure region just before the top of the hill.

d) Wind Tunnel Fringe Imaging Skin Friction (FISF) Tests. Figure 11 depicts results of the FISF testing; note the two local skin friction coefficient maxima, approximately halfway up the model, on either side of the model centerline. Note also the lower values of skin friction coefficient on the downstream side of the model; these roughly correspond to the region of separated flow indicated on previous oil flow images.

e) Wind Tunnel Cobra Probe Tests. Figures 12 and 13 depict results of the Cobra probe 3D velocity measurements. Figure 12 depicts the variation of centerline $(y=0.0)$ downstream velocity component, for both midpoint of the empty tunnel and for various locations upwind of the model. Figure 13 depicts several verticallateral planes of downstream Cobra measurements.

f) Wind Tunnel Particle Image Velocimetry (PIV) Tests. Figures 14 through 17 depict results of the PIV testing for the centerline plane $(y=0.0)$ of data. Figure 14 depicts the mean downstream velocity magnitude in color, with velocity vectors superimposed. Note the large region of separated and reversed flow downstream of the hill. Because the large number of vectors can tend to obscure color magnitudes, the remainder of the figures are 
shown with vectors suppressed. Figures 15 and 16 depict the unsteady streamwise velocity component and the uv Reynolds Stress component, respectively. Figure 17 depicts Turbulent Kinetic Energy (TKE).

\section{Summary}

A series of experimental tests, using both quantitative and qualitative techniques, were conducted at NASA Ames Research Center's Fluid Mechanics Lab (FML) to characterize both surface and flowfield wake characteristics of an axisymmetric, modified-cosine-shaped, wall-mounted hill. This initial report summarizes the experimental set-up, techniques used, and data acquired, and also describes some details of the dataset that is being constructed for use by other researchers, especially the CFD community. Subsequent reports will discuss the data and their interpretation in more detail.

\section{Acknowledgments}

This work was supported by the NASA Fundamental Aero Program (Subsonic Fixed Wing Project), with Dr. Mike Rogers, as DPMf for Efficient Aerodynamics. The authors also wish to thank Dennis Acosta, Louise Walker, Barry Porter, Laura Kushner, Ted Garbeff, and Murray Tobak, all of NASA Ames Research Center, as well as Summer interns, Katy Swanson (Rose-Hulman Institute of Technology) and Rob Bulmann (California Polytechnic University - San Luis Obispo) for their assistance in the completion of this project.

\section{References}

${ }^{1}$ Hunt, J.C.R., Abell, C.J., Peterka, J.A., Woo, H., "Kinematical Studies of the flows around free or surface-mounted obstacles; applying topology to flow visualization”, J. Fluid Mech. 86 (1), 1977, pages 179-200.

${ }^{2}$ Hunt, J. C. R. \& Snyder, W. H., "Experiments on stably and neutrally stratified flow over a model threedimensional hill”, J. Fluid Mech. 96, 1980, pages 671-704.

${ }^{3}$ Ishihara, T., Hibi, K., Oikawa, S., "A wind tunnel study of turbulent flow over a three-dimensional steep hill”, J. Wind Eng. Indus. Aerodyn. 83, 1999, pages 95-107.

${ }^{4}$ Apsley, D. D. \& Castro, I. P. "Flow and dispersion over hills: comparison between numerical predictions and experimental data", J. Wind Eng Ind. Aerodyn. 67-68, 1997, pages 375-386.

${ }^{5}$ Willits, S.M. \& Boger, D.A., "Measured and predicted flows behind a protuberance mounted on a flat plate" Applied Research Laboratory Report, Penn State Univ., State College, PA, August 30, 1999.

${ }^{6}$ Simpson, R.L., Long, C.H., and Byun, G. "Study of Vortical Separation From an Axisymmetric Hill”, International Journal of Heat and Fluid Flow 23, 2002, pages 582-591.

${ }^{7}$ Byun, G., Simpson, R. L. \& Long, C. H. "Study of vortical separation from three-dimensional symmetric bumps", AIAA J. 42 (4), 2004, pages 754-765

${ }^{8}$ Byun, G. \& Simpson, R. L. "Structure of three-dimensional separated flow on an axisymmetric bump", AIAA J. 44 (5), 2006, pages 999-1008.

'Byun, G. and Simpson, RL., "Surface-Pressure Fluctuations from Separated Flow over an Axisymmetric Bump", AIAA Journal Vol. 48, No. 10, October 2010

${ }^{10}$ Thiele, F. \& Jakirli, S., "12 ${ }^{\mathrm{TH}}$ ERCOFTAC/IAHR/COST Workshop on Refined Turbulence Modelling”, Technical University of Berlin, Germany, October 12-13, 2006.

${ }^{11}$ Patel, N. and Menon, S., "Structure of flow separation and reattachment behind an axisymmetric hill", Journal of Turbulence Volume 8, 2007.

${ }^{12}$ Persson, T., Liefvendahl, M., Bensow, R.E., \& Fureby, C.,"Numerical investigation of the flow over an axisymmetric hill using LES, DES, and RANS", Journal of Turbulence, Volume 7, 2006.

${ }^{13}$ Krajnovic, S., "Large Eddy Simulation of the Flow Over a Three-Dimensional Hill" Flow, Turbulence and Combustion Volume 81, Numbers 1-2, 2008, pages 189-204.

${ }^{14}$ Garcia-Villalba, M, Li, N., Rodi, W., and Leschziner, M.A., “ Large-eddy simulation of separated flow over a three-dimensional axisymmetric hill”, J. Fluid Mech. vol. 627, 2009, pp. 55-96.

6

American Institute of Aeronautics and Astronautics 


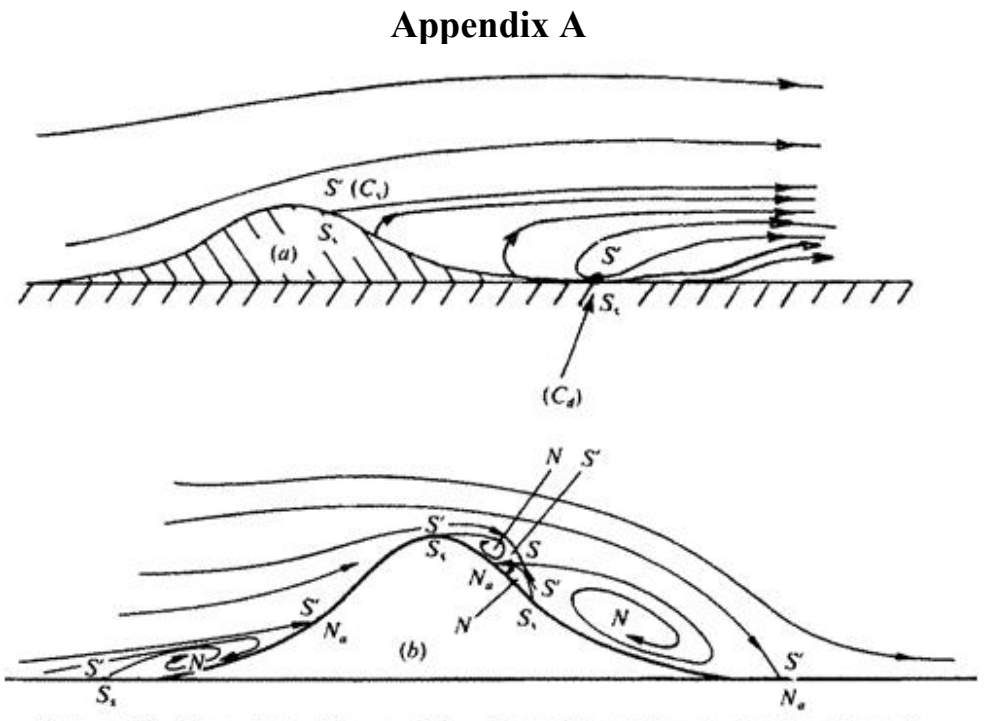

Froure 16. Mean streamlines and singular points on the centre-line plane of the flow over axisymmetric humps. (a) Laminar flow. (b) Turbulent flow.

Figure 1 Flow Over a Wall-Mounted Axisymmetric Hill (From reference 1)
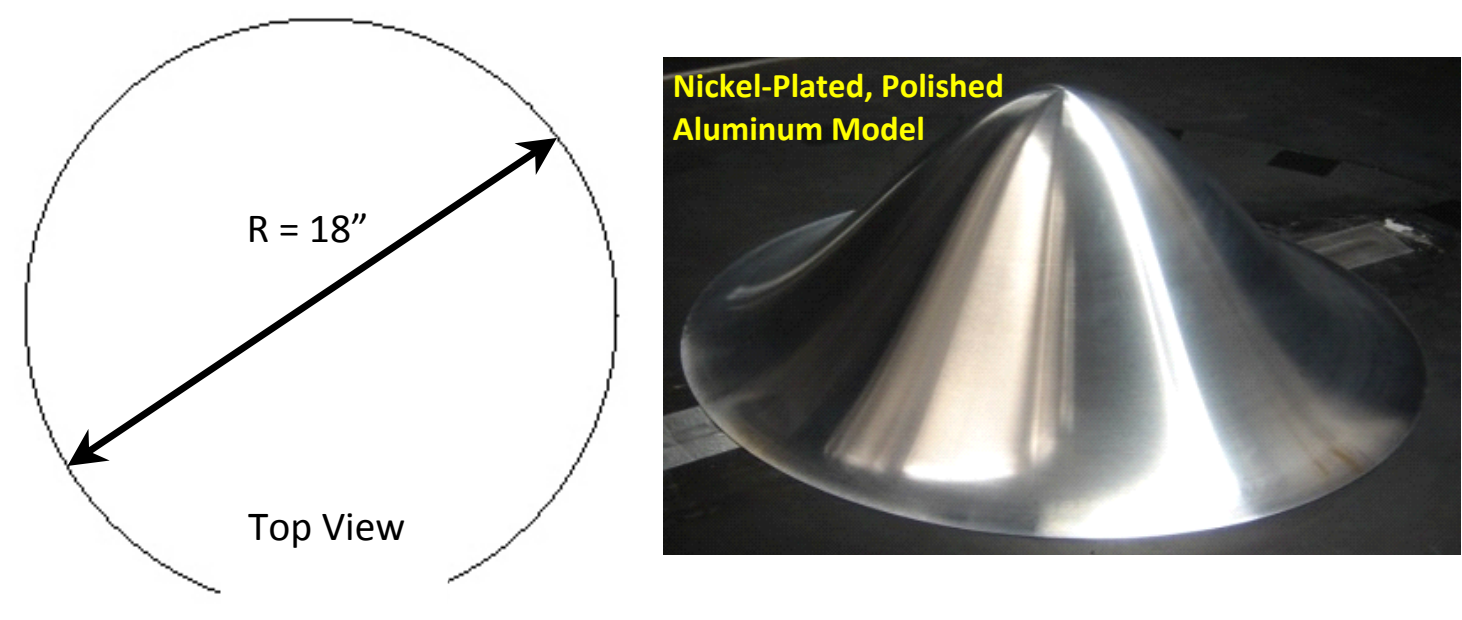

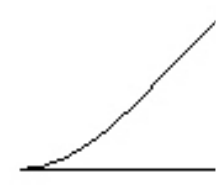

Front View

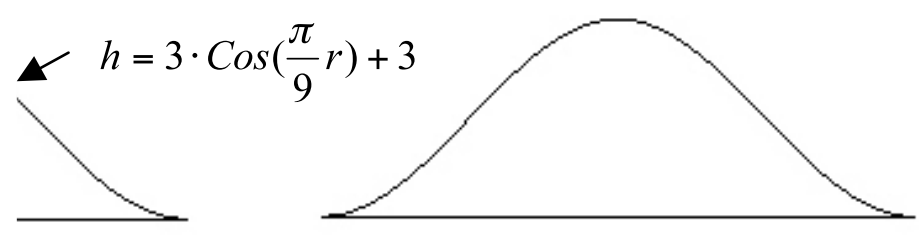

Side View

Figure 2 Aluminum Wind Tunnel Mor 


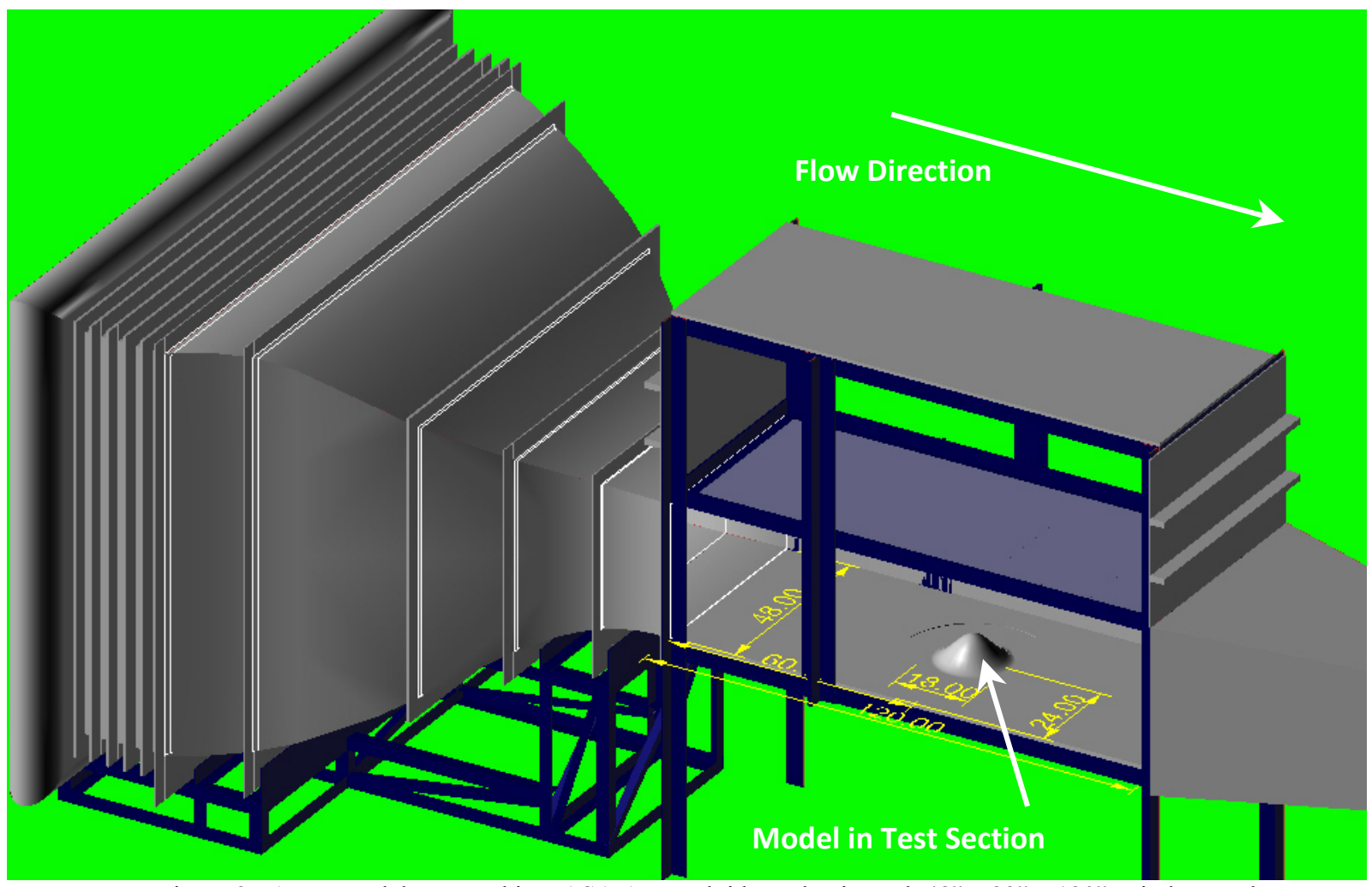

Figure 3 FAITH model mounted in NASA Ames Fluid Mechanics Lab 48” x 32" x 120” Wind Tunnel

Overall Facility Dimensions: $17.0^{\prime} \mathrm{L} x 7.5^{\prime} \mathrm{W} \times 4.5^{\prime} \mathrm{H}$

Facility Operating Capacity: 800 gal max)

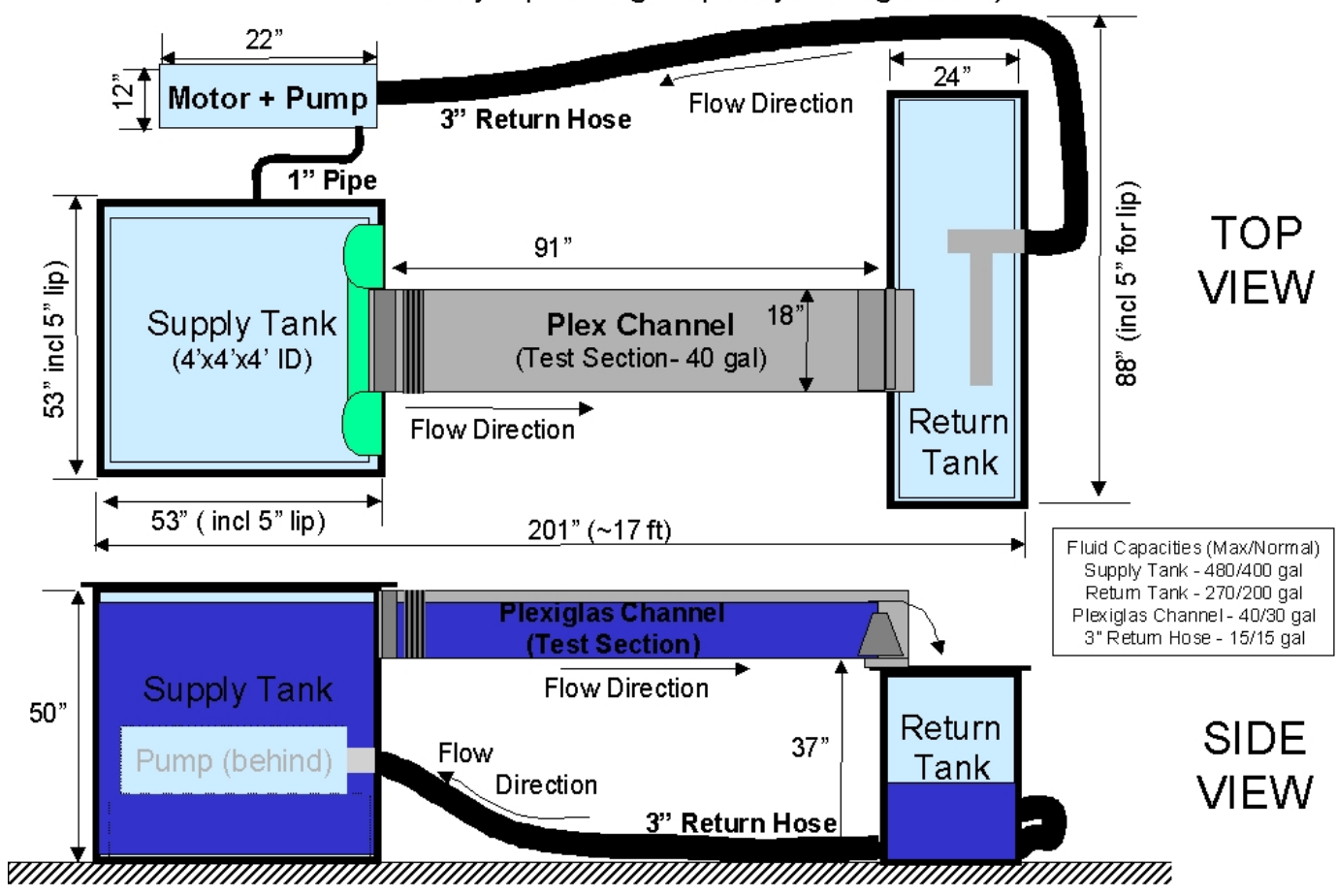

Figure 4 NASA Ames Fluid Mechanics Lab Water Channel

8

American Institute of Aeronautics and Astronautics 


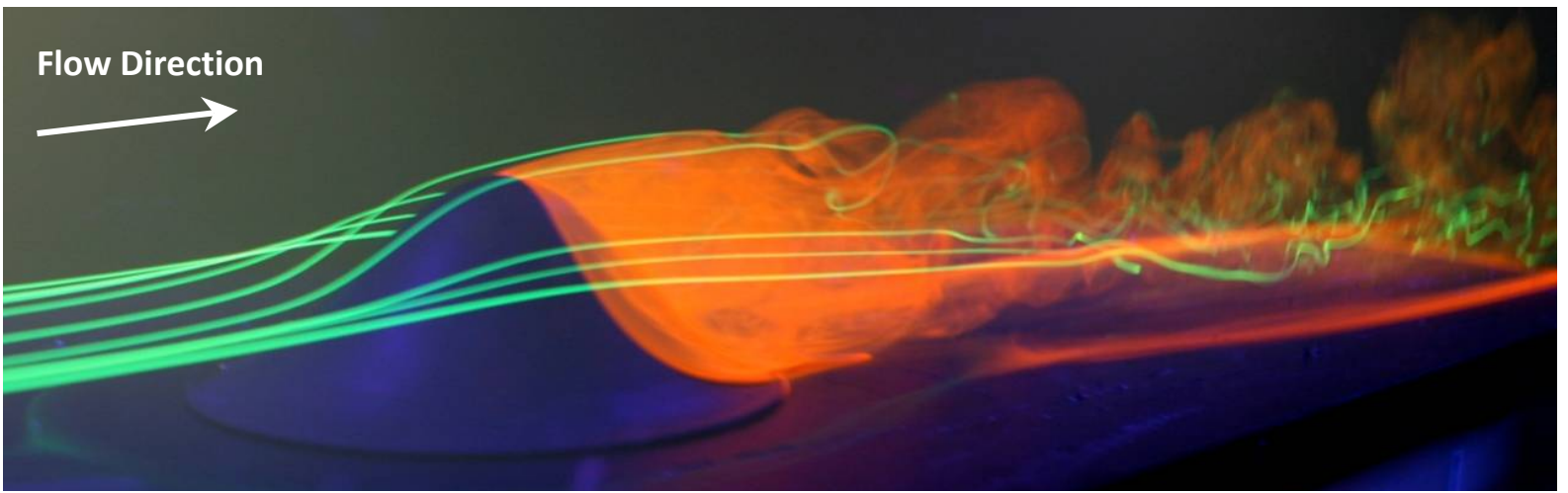

Figure 5 FAITH Water Channel Flow Visualization (View from Upstream)

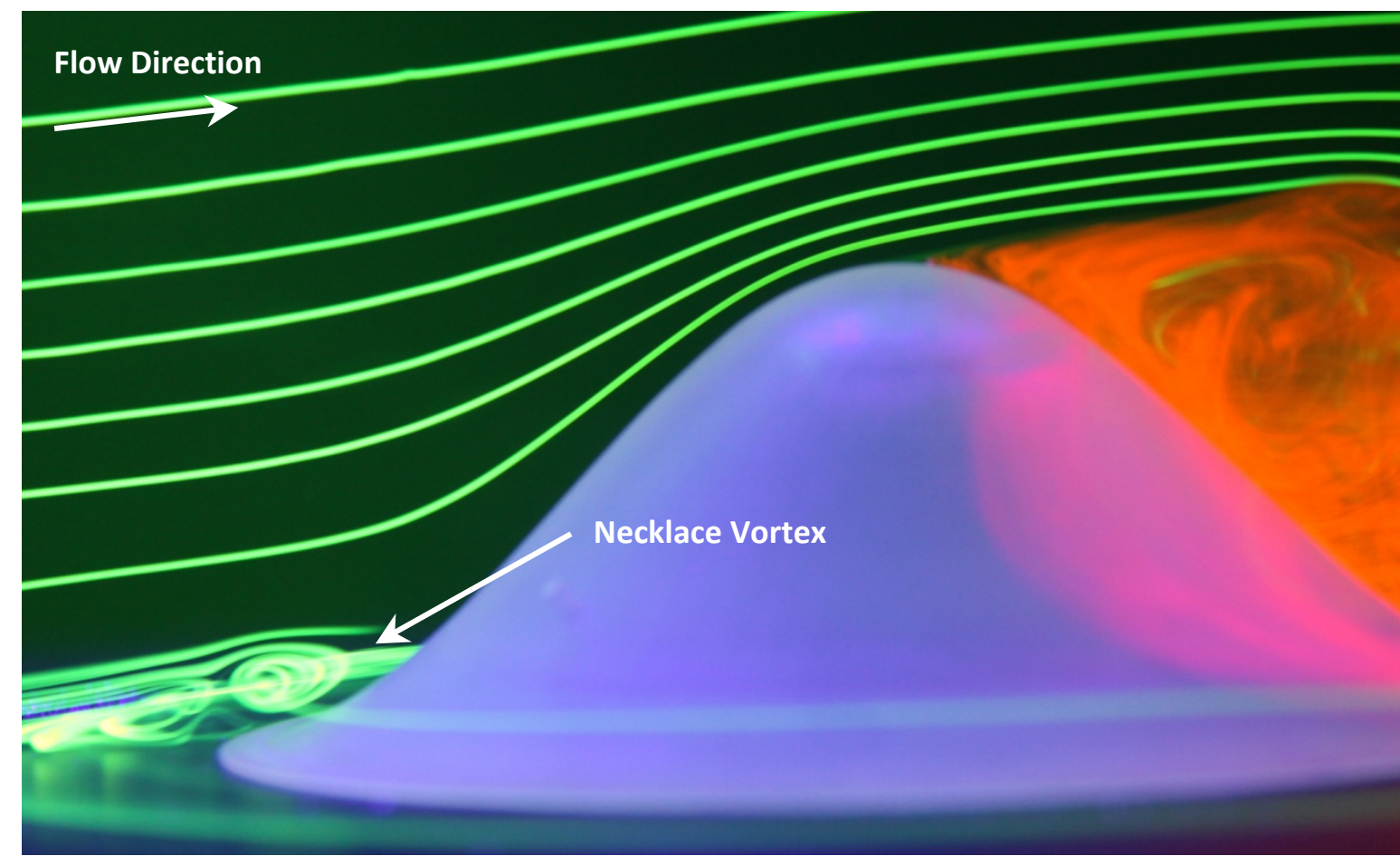

Figure 6 FAITH Water Channel Flow Visualization (showing upstream necklace vortex)

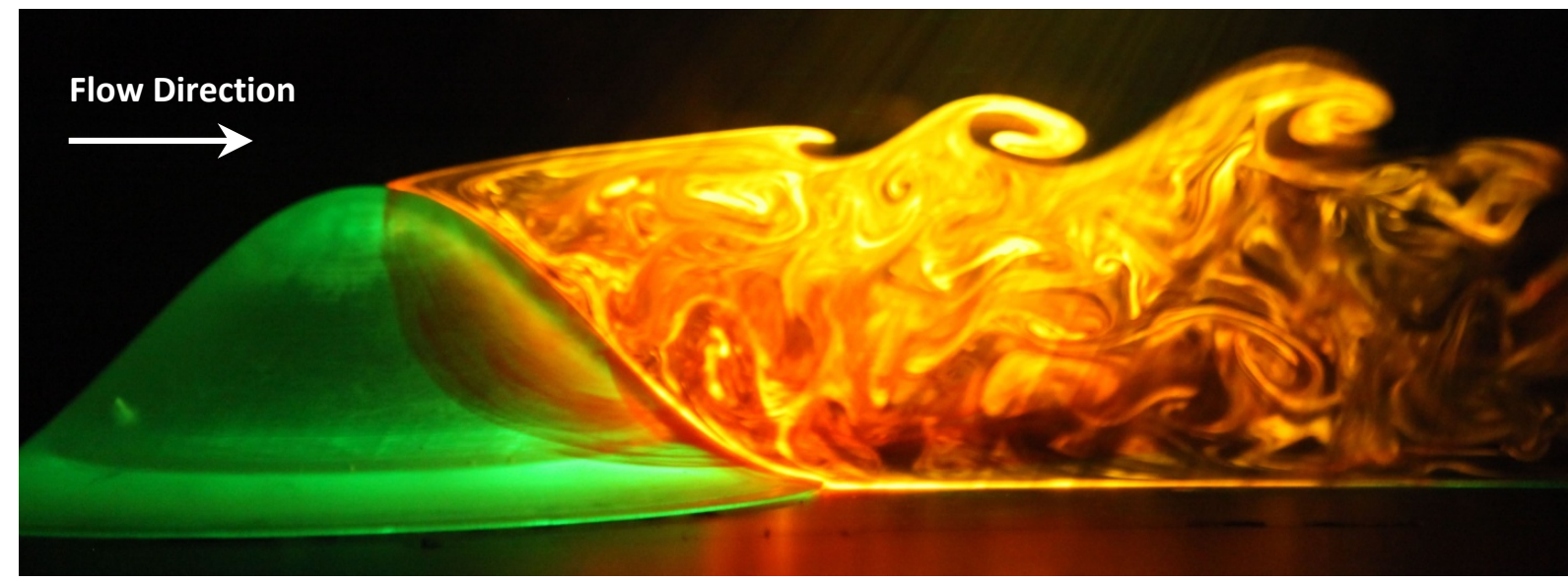

Figure 7 FAITH Water Channel Flow Visualization (Laser sheet centerline illumination)

American Institute of Aeronautics and Astronautics 


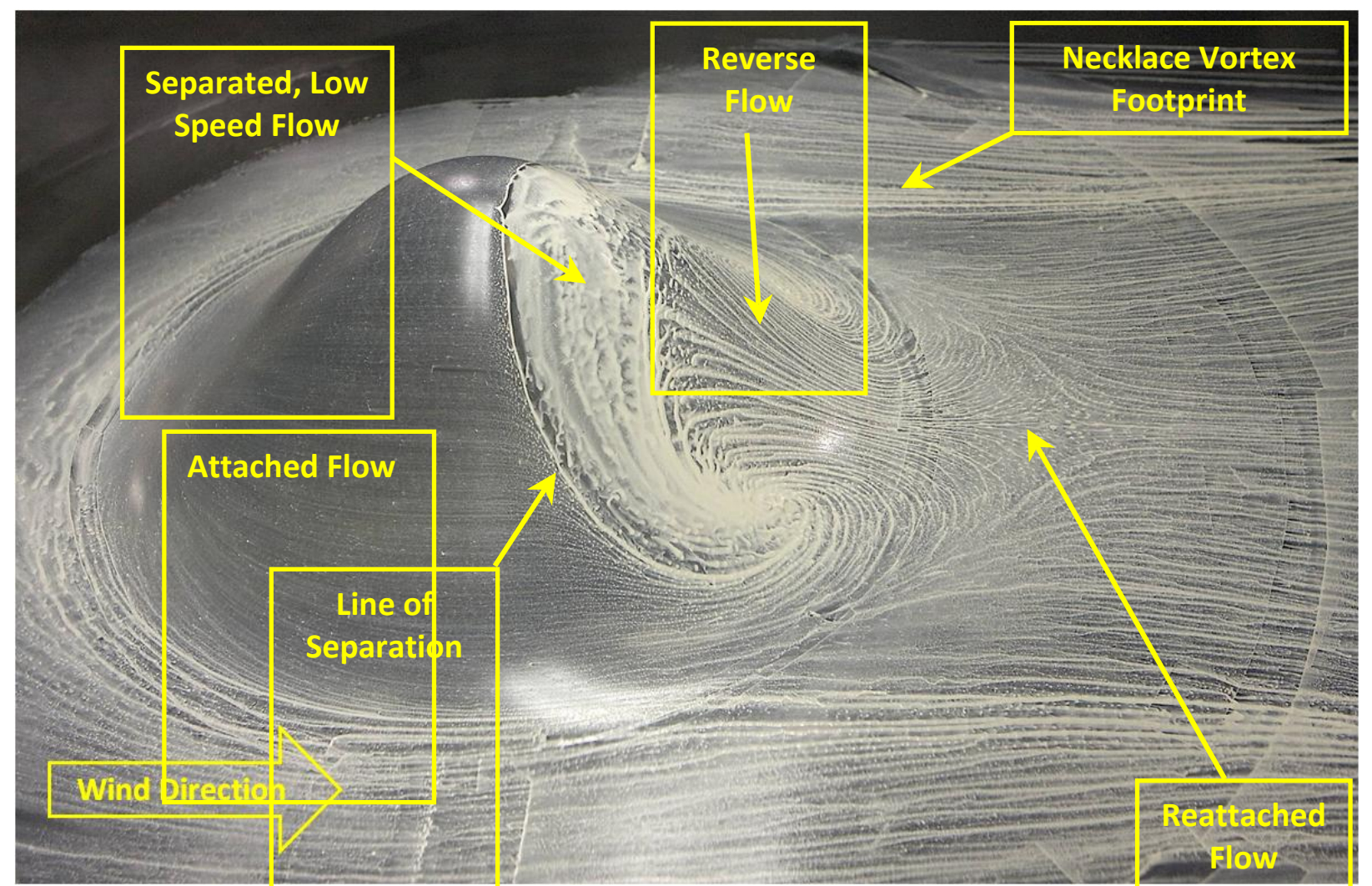

Figure 8 FAI'TH Wind Tunnel Oil Flow Visualization Experiments

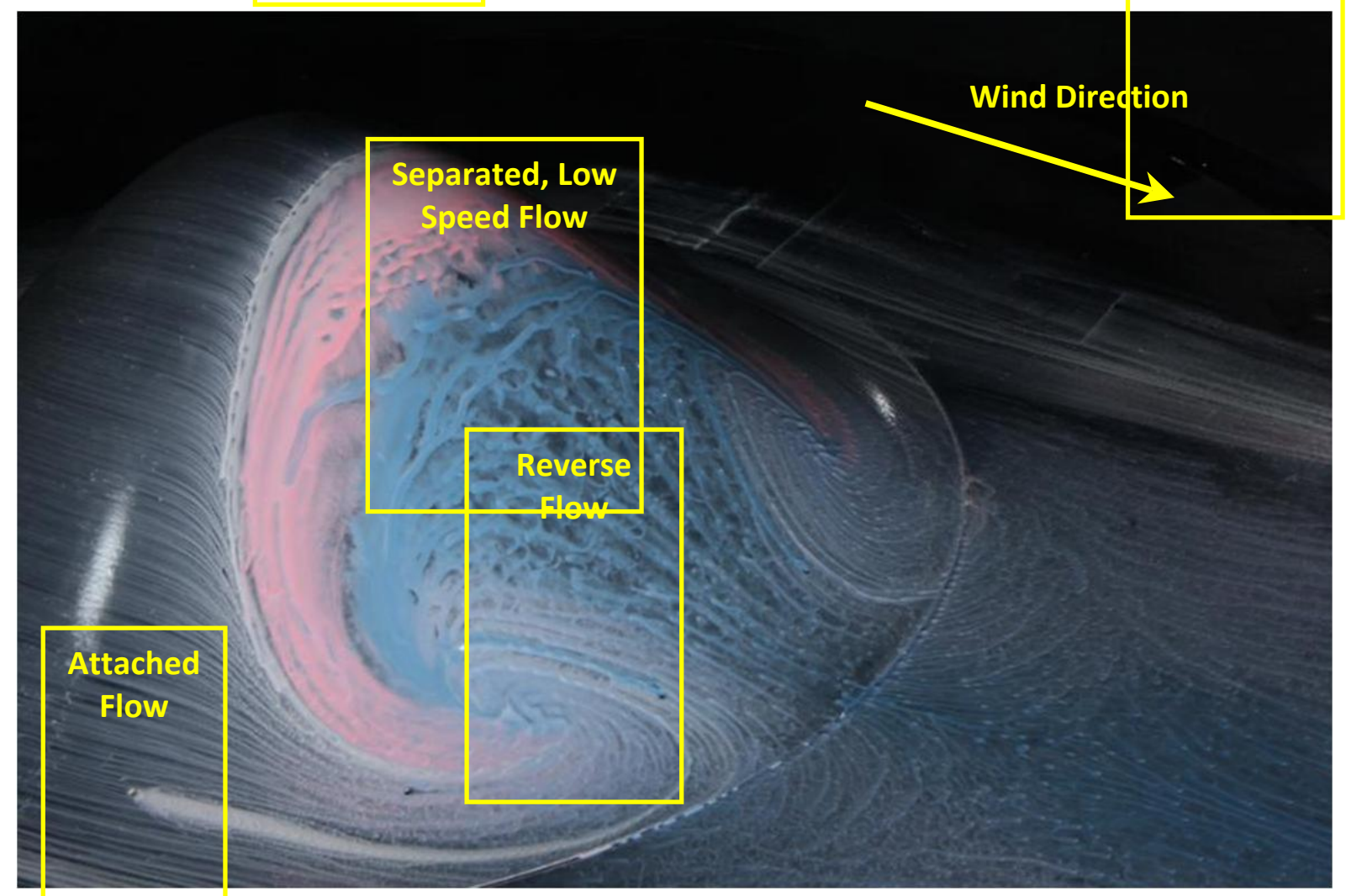

]igure 9 FAITH Wind Tunnel Oil Flow Visualization Experiments (white, pink, and blue) 


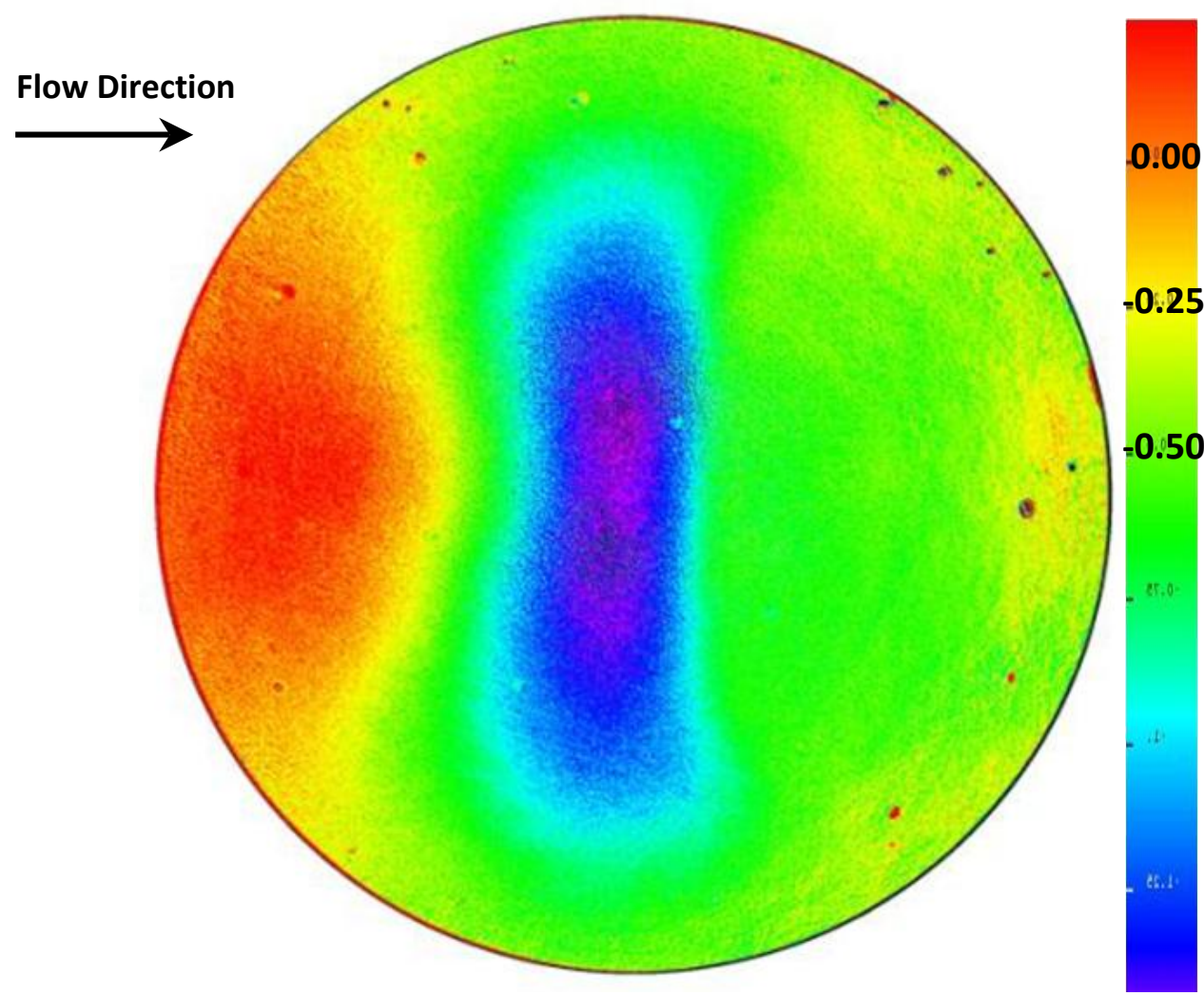

Figure 10 FAITH PSP Experiment Data: Mean Surface Pressure Coefficient, Cp

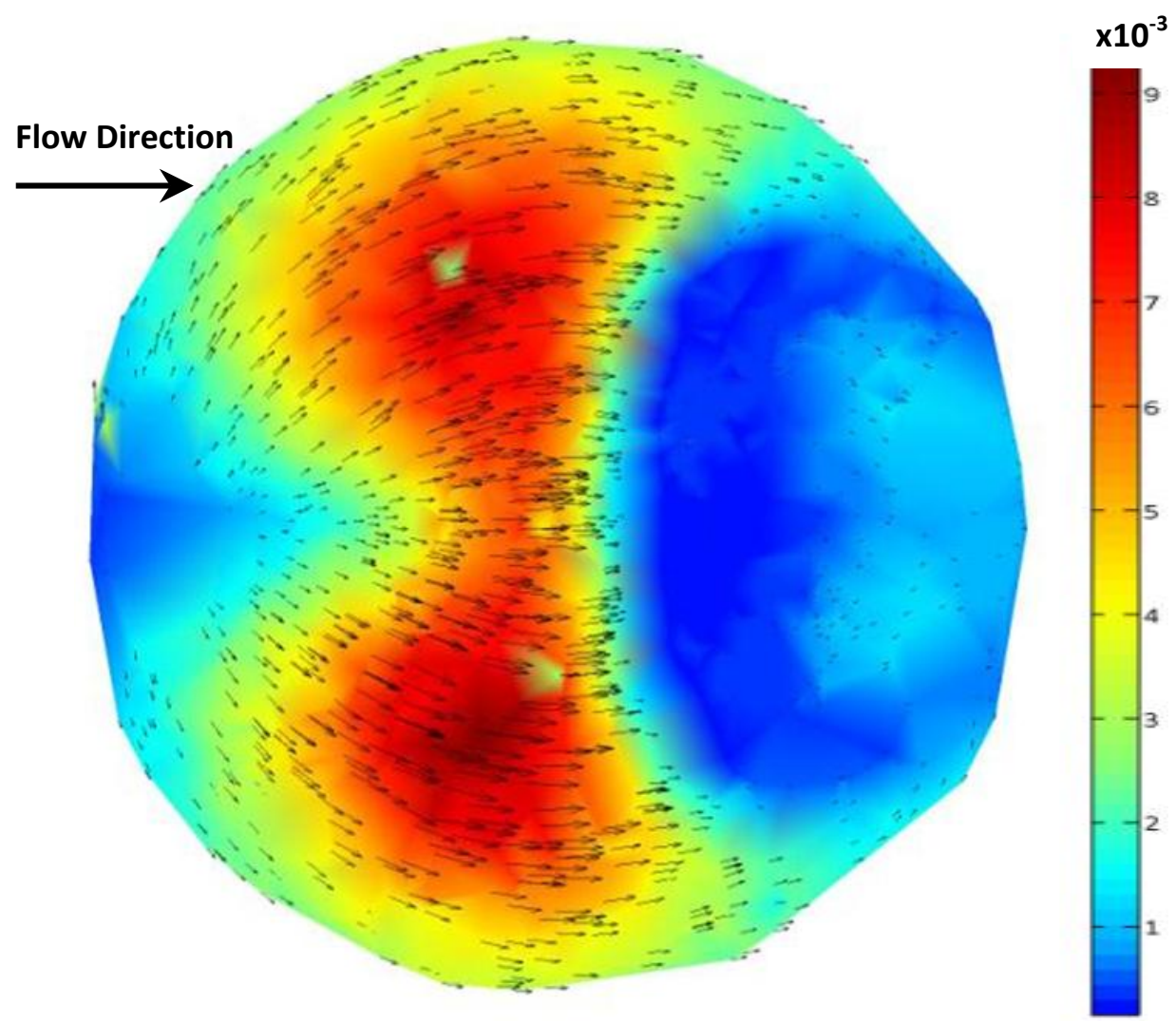

Figure 11 FAITH FISF Data: Skin Friction Coefficient $\left(\mathrm{C}_{\mathrm{f}}\right)$ Magnitude and Skin Friction Direction 11 American Institute of Aeronautics and Astronautics 


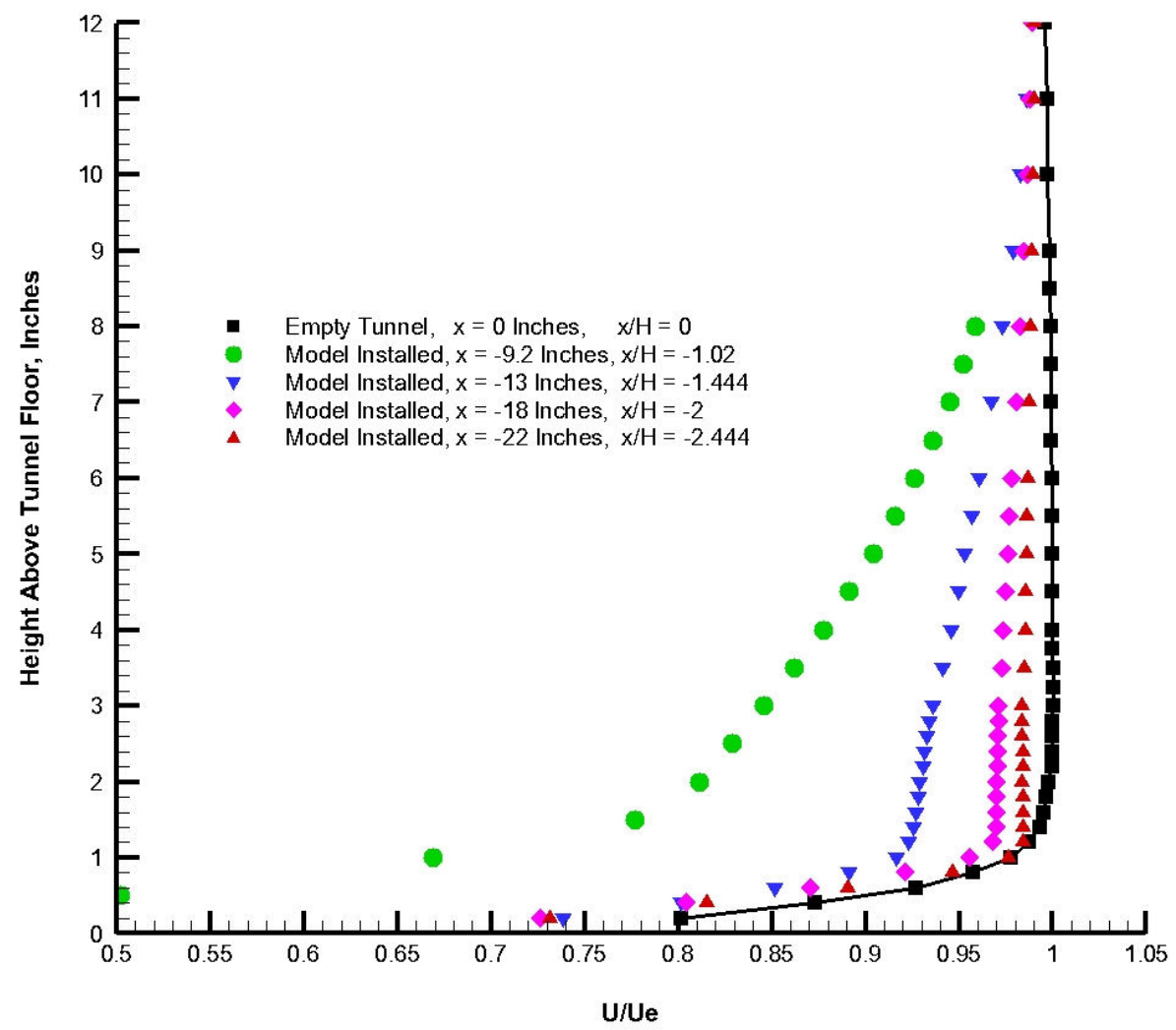

Figure 12 Cobra Probe Mean Centerline $(\mathrm{y}=0.0)$ Velocity at Various Distances Upwind of Model Centroid

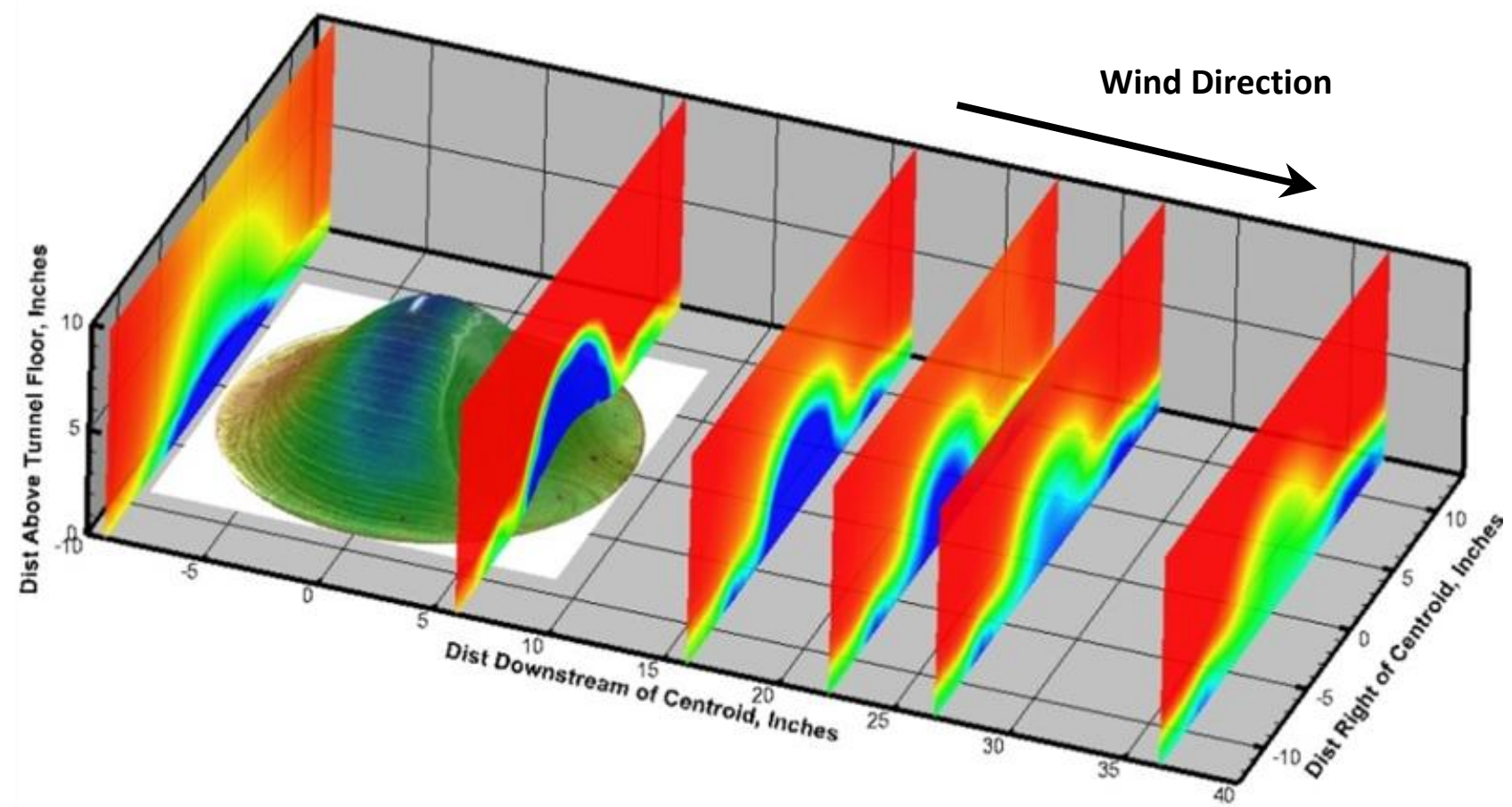

Figure 13 Combined Data: PSP, Oil Flow, and Cobra Probe Wake Measurements

American Institute of Aeronautics and Astronautics 


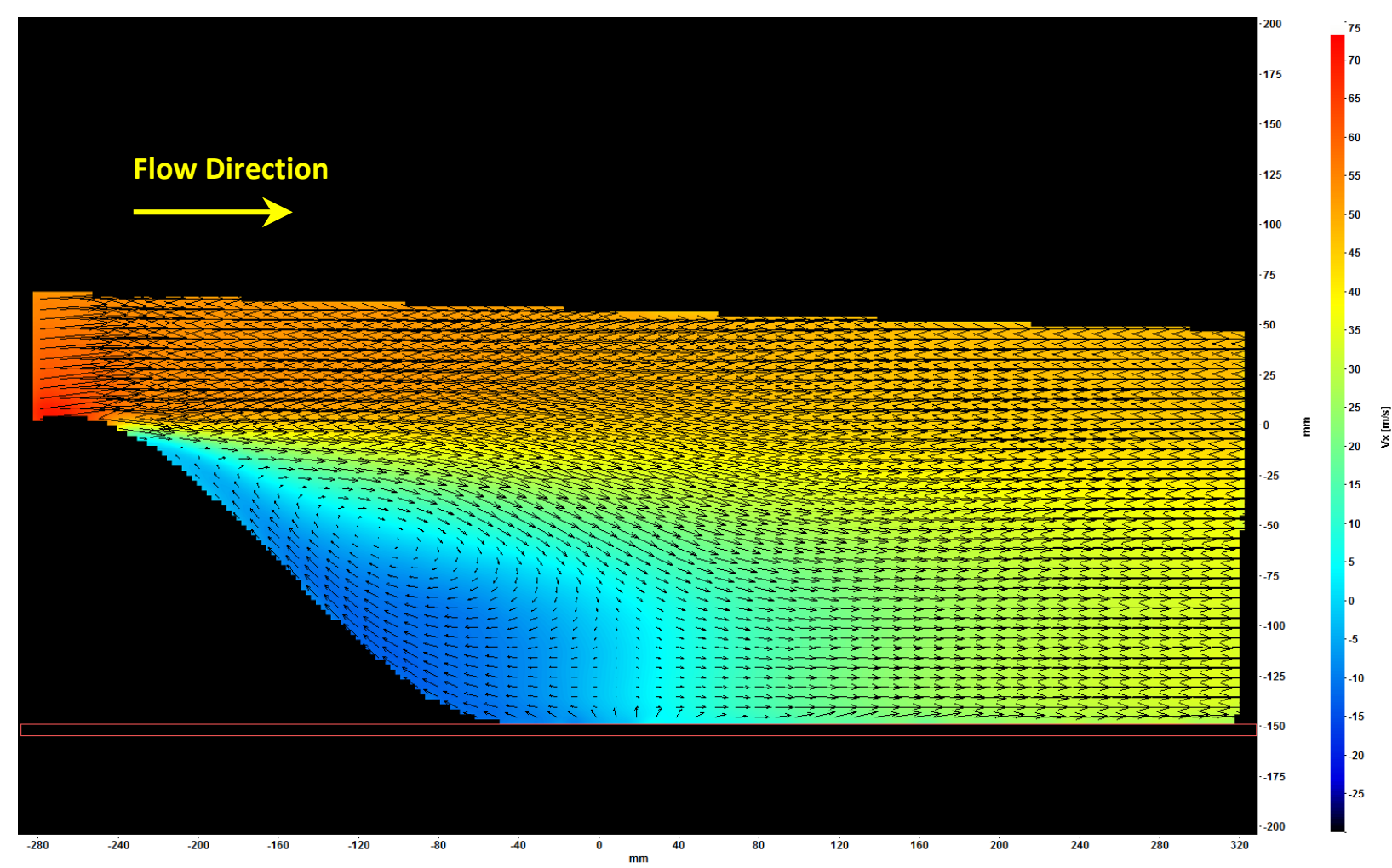

Figure 14 FAITH PIV data (Time-averaged downstream velocity ( ) magnitude, in-plane velocity vectors)

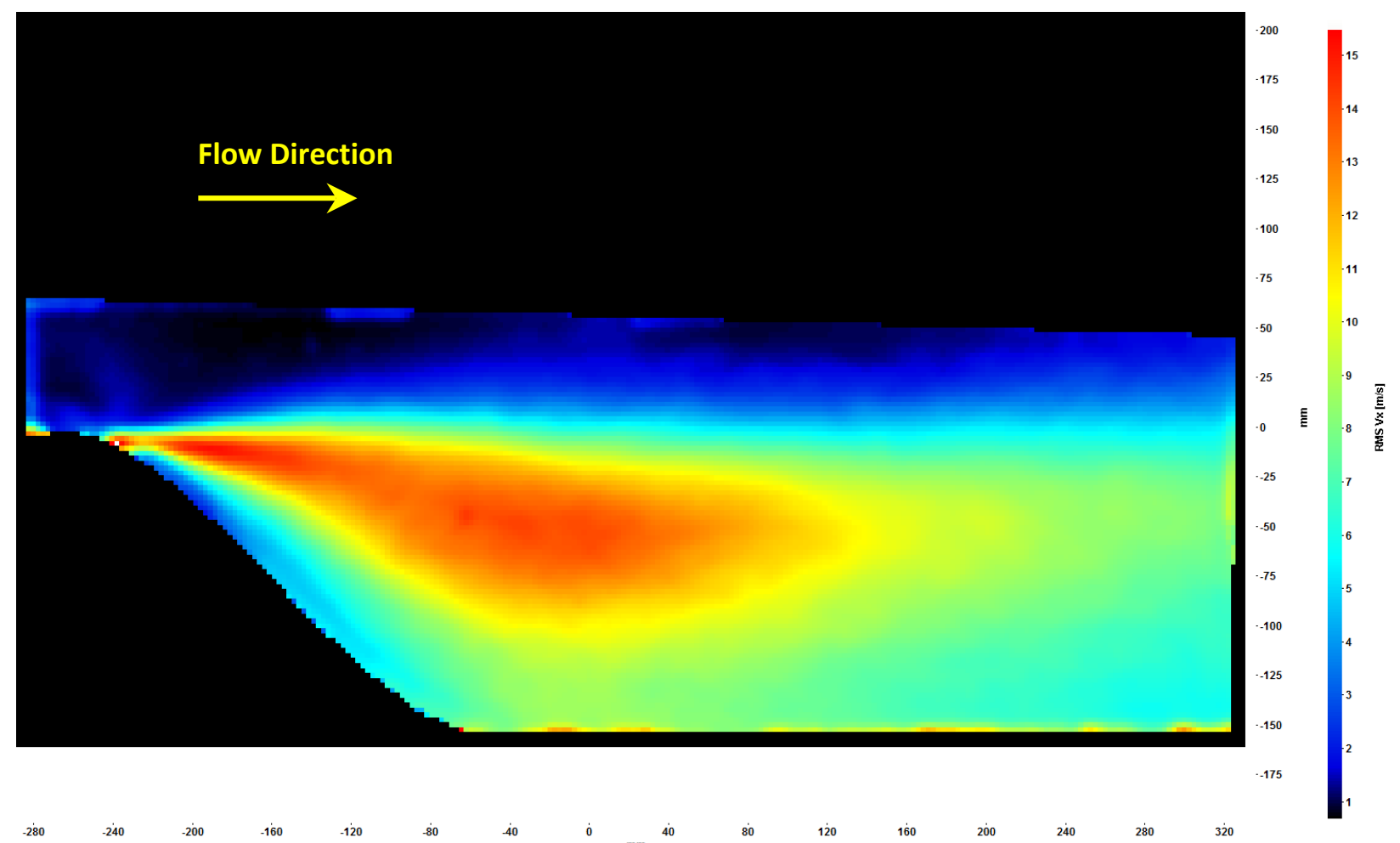

Figure 15 FAITH PIV unsteady downstream velocity data, ( ')2

American Institute of Aeronautics and Astronautics 


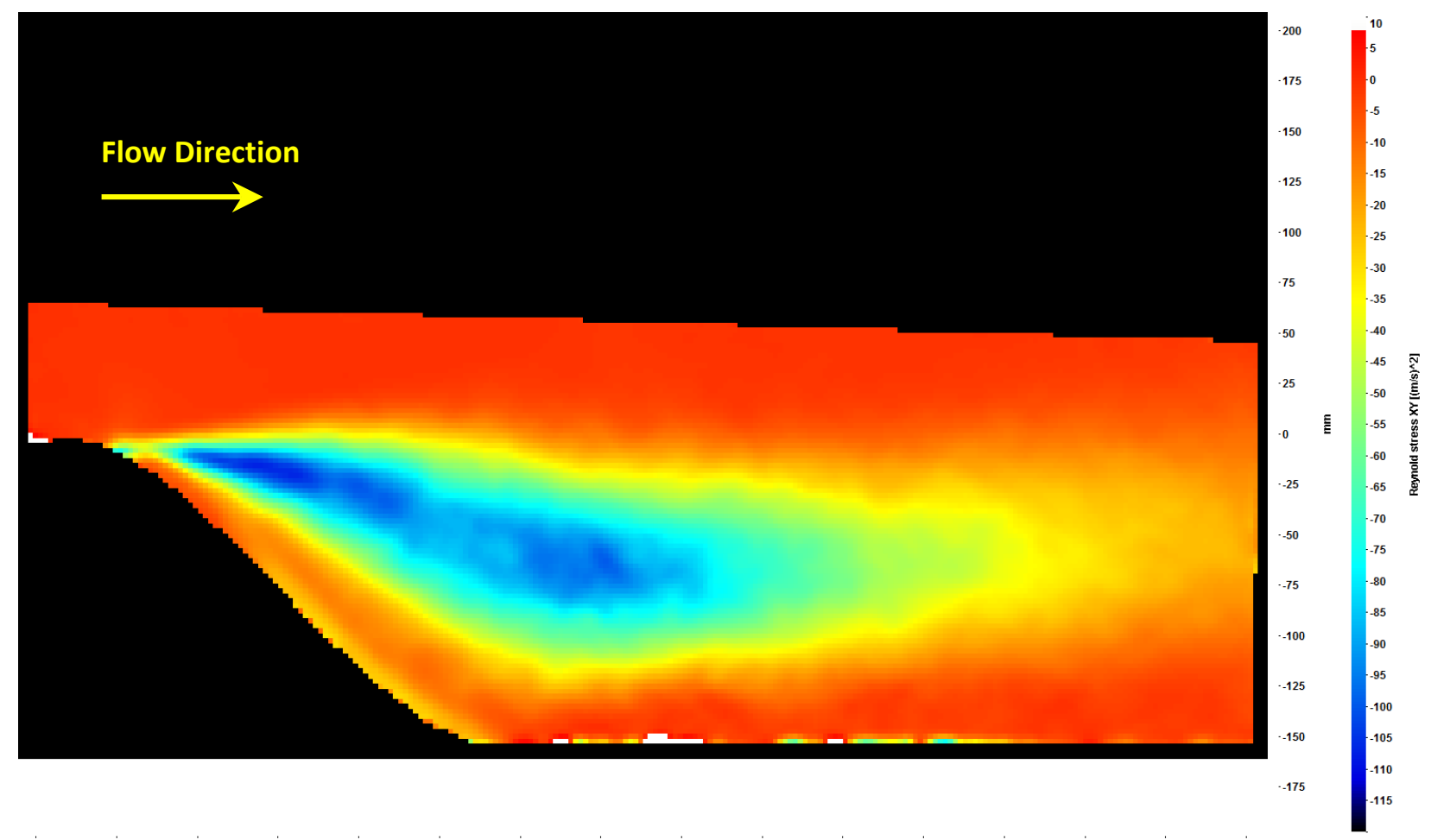

Figure 16 FAITH PIV Reynolds Stress data ( ' ' )

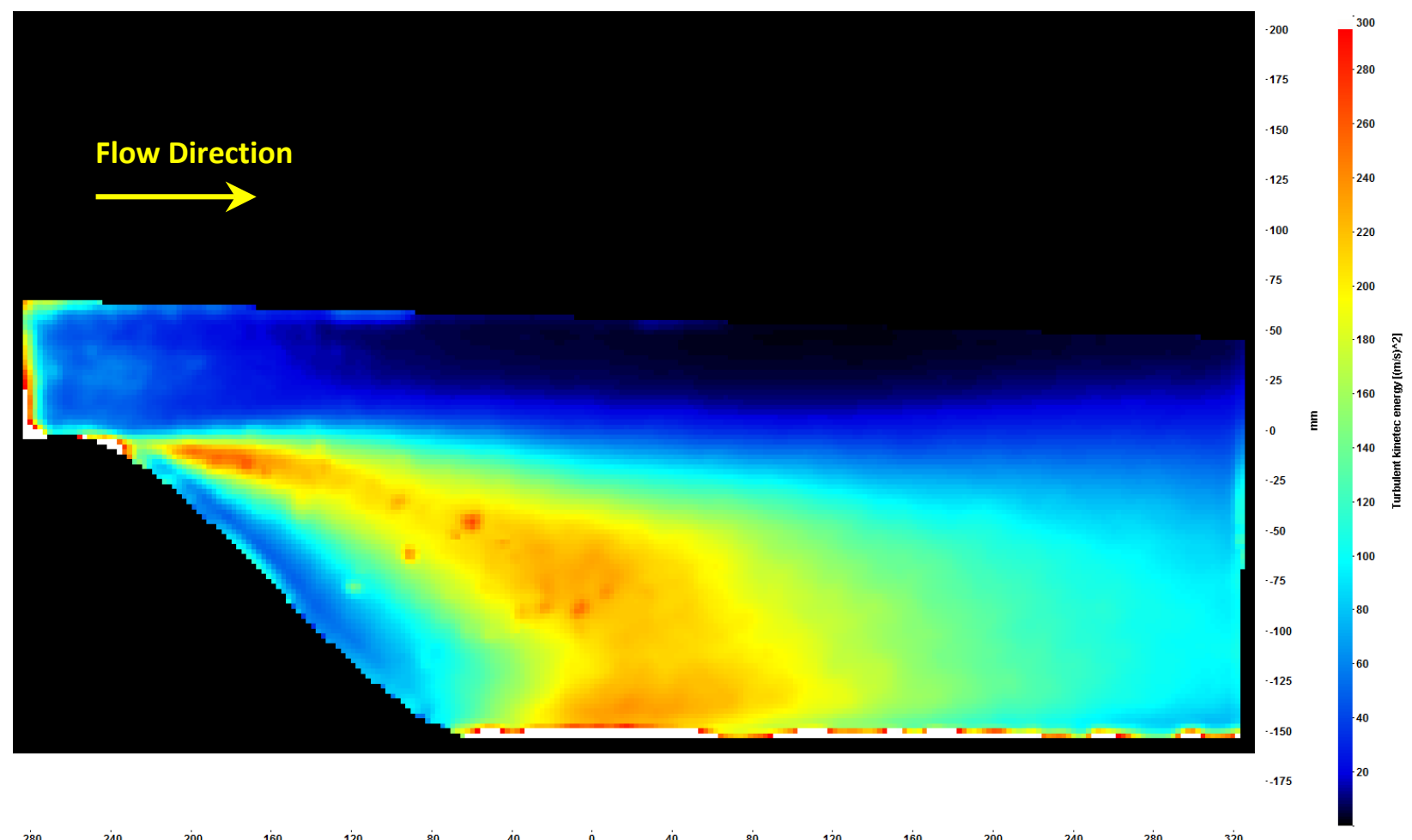

Figure 17 FAITH PIV data (TKE) 